\title{
Multiobjective Contactless Delivery on Medical Supplies under Open-Loop Distribution
}

\author{
Huilin Li $\mathbb{D}$, Ke Xiong, and Xiuming Xie \\ Southern University of Science and Technology Yantian Hospital, Shenzhen 518081, Guangdong, China \\ Correspondence should be addressed to Huilin Li; 1228587935@qq.com
}

Received 28 March 2021; Revised 24 April 2021; Accepted 16 May 2021; Published 15 June 2021

Academic Editor: Fuli Zhou

Copyright (c) 2021 Huilin Li et al. This is an open access article distributed under the Creative Commons Attribution License, which permits unrestricted use, distribution, and reproduction in any medium, provided the original work is properly cited.

\begin{abstract}
With the development and popularity of intelligent store terminals, contactless distribution has been a hot talk on medical supplies using intelligent express boxes. Based on the traditional vehicle routing problem, this paper considers the sharing economy and open-loop distribution reality hotspots and considers the optimization of carbon emissions in contactless distribution. The travel distance and load capacity are the key factors affecting carbon emissions. The carbon emission model proposes dual goals of minimizing distribution costs and carbon emissions. It constructs a distribution path planning model with multiple distribution stations. To solve this problem with multidepot optimization, we design a hybrid genetic algorithm, and according to the strategy of customer distance clustering analysis, the dispatching of vehicles is divided into three steps. The principle of elite crossing is applied to avoid the solution to fall into local optimum. The experimental results show that the proposed model and optimization algorithm can get a tradeoff between the logistics cost and carbon emissions.
\end{abstract}

\section{Introduction}

To solve the problems of fast allocation of medical supplies in the prevention of COVID-19, employing external vehicles to realize logistics distribution has become an important strategy and measure under the background of sharing economy. In addition, contactless terminal equipment (such as self-pickup boxes) also is applied for receiving medical supplies to avoid infection [1]. Finally, the distribution vehicle does not have to return to the original distribution center after completing the distribution task, it can continue to serve other distribution center or demand points, so the distribution problem can be simulated as an open-vehicle routing problem (OVRP), which not only reduces delivery time significantly but also ensures the safety of distribution in COVID-19 [1, 2].

In medical supplies, Liu et al. focus on integrated planning for public health emergencies and build a modified model for controlling H1N1 pandemic [3]. Garza-Reyes et al. focus on the improvement of transport and logistics performance of the medical service system; they build mathematical models to optimize medical supplies distribution [4]. Zhang et al. reveal the critical role of timely supply of medical resources to avoid great infection [5]. Zhou et al. and He et al. focus on the semantic match of medical supplies to transport the medical resources to required places in an efficient way; this paper studies the medical transportation resource discovery mechanism, leading to efficiency improvement and operational innovation [6-8].

At present, in logistics distribution, most research on OVRP mainly focuses on reducing logistics costs or reducing the number of vehicles used [9-12]. With people's attention to energy conservation, emission reduction, and green logistics, how to protect the environment while reducing logistics costs has become a hot issue in academia and environmental protection departments. Bektas and Laporte [13] empirically studied the influencing factors of carbon emissions and weighed the relationship between the driving distance, driver's salary, and fuel consumption. Xiao et al. [14] described the carbon emission in the process of logistics distribution from a microperspective and compared the distribution routes under three different target situations: carbon emission, logistics distribution cost, and total 
distribution distance. Considering the impact of road congestion on carbon emissions, Woensel et al. [15] compared and analyzed the carbon emissions under different driving speeds. Demir et al. [16] proposed that vehicle load, engine type and size, road slope, and other factors have a certain relationship with fuel consumption of distribution vehicles and further affect carbon emissions. Niu et al. [17] considered carbon emission cost into logistics cost, established a green open-loop vehicle routing problem, and designed a hybrid tabu search algorithm to solve the model.

From the existing research point of view, the research on the vehicle routing problem of minimizing carbon emissions mostly considers the case of a single distribution station, while the research on the vehicle routing problem of the logistics distribution system with multiple distribution stations is less. At the same time, in the study of the impact of carbon emissions on the logistics distribution system, the vast majority of research is still based on the weighted sum of single-objective optimization, and the research on the vehicle routing problem of the logistics distribution system is not enough. Carbon emissions multiplied by the carbon price coefficient and weighted to the logistics cost, in essence, is still to minimize the logistics cost as the goal and get a single decision-making result. In practical application, decision makers often need to obtain an optimal solution set and select a satisfactory logistics distribution scheme according to their preferences. Therefore, it is more practical to study the multiobjective open-loop vehicle routing problem with the objective of minimizing carbon emissions and logistics costs in the case of multiple distribution stations. Considering that the carbon emission of vehicles has a positive correlation with mileage and actual load $[18,19]$, an optimization model of carbon emission is established, a multiobjective multidistribution station vehicle routing problem is proposed to minimize logistics cost and carbon emission, and a mixed-integer programming model of the problem is established. Furthermore, a multiobjective genetic algorithm based on decomposition is designed to solve the problem. The simulation results show that the algorithm can solve the problem effectively.

\section{Problem Description and Model}

A city logistics distribution system has $m$ distribution centers and $n$ consumers. Considering the constraints of vehicle fuel consumption and the driver's workload, the maximum carrying capacity of the distribution vehicle is $Q$, and the driving distance of the distribution vehicle is not more than $L$. In order to minimize the logistics cost and carbon emissions of vehicle distribution, it is required to arrange the distribution vehicles and their driving paths reasonably under the constraints of vehicle load and driving distance. A feasible distribution scheme needs to meet the following conditions:

(1) The number of vehicles in each distribution center is limited, and the vehicles are rental vehicles

(2) Vehicles can only start from the distribution center and deliver the loaded goods to the designated customers along a distribution route without returning to the original distribution center

(3) One delivery vehicle can serve multiple customers, but each customer is only served once by one vehicle

(4) Each vehicle has the constraints of capacity and maximum driving distance, and all distribution vehicles are homogeneous

In order to establish the mathematical model of the problem, the symbols are defined as follows:

2.1. Symbol Definition. In this section, we describe some symbols used in this model.

(1) Sets:

$C$ : consumer set, $C=\left\{c_{1}, c_{2}, \ldots, c_{n}\right\}$, the number of $N$ consumers

$D$ : distribution center set, $D=\left\{d_{1}, d_{2}, \ldots, d_{m}\right\}$, the number of $M$ distribution stations

$N$ : distribution station and consumer set, $N=C \cup D$

$K$ : distribution vehicle set, $K=\left\{k_{1}, k_{2}, \ldots, k_{m}\right\}$

(2) Parameters:

$q_{i}$ : demand for consumer $i\left(0 \leq q_{i} \leq Q\right)$

$d_{i j}$ : Euclidean distance between any two points $i$ and $j, i, j=1,2, \ldots, n, \ldots, m+n$

$\omega$ : empty net weight of delivery vehicle

Q: maximum loading capacity of distribution vehicles

$L$ : maximum distance of distribution vehicles

$C_{f}$ : rental fee per delivery vehicle

$C_{v}$ : variable cost of vehicle unit (including workers' wages, vehicle daily maintenance fees, etc.)

(3) Decision variables:

$x_{i j k}$ : if the vehicle $k$ goes arc $(i, j)$; otherwise, it is 0 $f_{i j k}$ : the cargo loading capacity of distribution vehicle $k$ going by $(i, j)$

2.2. Description Method of Vehicle Carbon Emission. Existing research results show that there is a positive correlation between vehicle carbon emissions and fuel consumption, so fuel consumption can be used to describe its carbon emissions $[20,21]$. Considering the relationship between the actual load and distance of the vehicle and the fuel consumption, the paper determines using the following method to calculate the fuel consumption:

$$
F_{i j}=\left(\rho^{0}+\frac{\rho^{*}-\rho^{0}}{Q} y_{i j}\right) d_{i j},
$$

where $\rho^{*}$ and $\rho^{0}$ are the fuel consumption rates of full-load and no-load vehicles, respectively, and $F_{i j}$ represents the fuel consumption of vehicles passing through the path. Therefore, according to the relationship between carbon emissions and fuel consumption, the calculation method of vehicle carbon emissions is as follows: 


$$
\mathrm{EM}_{i j}=\mathrm{FE} \cdot F_{i j}
$$

where FE represents the carbon emissions per unit of fuel consumption.

2.3. Multiobjective Multidepot OVRP. According to the abovementioned symbol definition and carbon emission calculation method, the multidepot open vehicle routing problem (MDOVRP) model for minimizing logistics cost and carbon emission is as follows:

$$
\begin{aligned}
& \operatorname{Min}_{f} \sum_{k \in K} \sum_{i \in D} \sum_{j \in C} x_{i j k}+C_{v} \sum_{k \in K} \sum_{i \in N} \sum_{j \in N} x_{i j k} d_{i j}, \\
& \operatorname{Min~FE} \sum_{k \in K} \sum_{i \in N} \sum_{j \in N} x_{i j k} d_{i j}\left(\rho^{0}+\frac{\rho^{*}-\rho^{0}}{Q} y_{i j}\right),
\end{aligned}
$$

subject to

$$
\begin{gathered}
\sum_{k \in K} \sum_{i \in N} x_{i j k}=1, \quad \forall j \in C, \\
\sum_{k \in K} \sum_{j \in N} x_{i j k}=1, \quad \forall i \in C, \\
\sum_{i \in N} x_{i h k}-\sum_{j \in N} x_{h j k}=0, \quad \forall k \in K ; h \in N, \\
\sum_{i \in N} q_{i} \sum_{j \in N} x_{i j k} \leq Q, \quad \forall k \in K, \\
\sum_{i \in N} \sum_{j \in N} d_{i j} x_{i j k} \leq L, \quad \forall k \in K, \\
\sum_{i \in D} \sum_{j \in C} x_{i j k} \leq 1, \quad \forall k \in K, \\
\sum_{j \in D} \sum_{i \in C} x_{i j k} \leq 1, \quad \forall k \in K, \\
\sum_{k \in K} q_{j} x_{i j k} \leq \sum_{k \in K} f_{i j k}, \quad \forall i \in N, \forall j \in D, \\
\sum_{i \in N} f_{j i k}=q_{j}, \quad \forall j \in N, \forall k \in K,
\end{gathered}
$$

The objective function in eq. (3) represents the minimum logistics distribution cost, including the rental cost and variable cost of distribution vehicles. The objective function in eq. (4) represents the minimum carbon emission. The constraints in eqs. (5) and (6) indicate that each consumer is only served once by only one vehicle. The constraint in eq. (7) ensures the continuity of vehicle driving. The constraints in eqs. (8) and (9), respectively, indicate the capacity constraints and mileage constraints of vehicles. The constraints in eqs. (10) and (11) indicate the availability constraints of vehicles, that is, determining the vehicle's driving capacity whether it is used or not. The constraints in eqs. (12)-(14) indicate the load limit of the vehicle driving on the side. The constraint in eq. (15) indicates the $0-1$ variables.

\section{Algorithm Design}

Genetic algorithm is a population-based search algorithm, which can get multiple nondominated solutions in one run. It is widely used in NP-hard problems such as VRP [22, 23]. The algorithm designed in this paper is as follows.

3.1. Decomposition of Multiobjective Optimization Problems. The decomposition-based multiobjective optimization algorithm decomposes the multiobjective optimization problem into several single-objective subproblems to solve. The weighted sum method and Chebyshev method are the commonly used decomposition methods. The weighted sum method depends on the characteristics of the problem and is not suitable for solving the optimization problem with a nonconvex front. Therefore, this paper uses the Chebyshev method to solve the multiobjective problem line decomposition.

3.2. Expression of Solution. Considering the coding characteristics of the MDVRP, natural number coding is adopted to avoid the conversion between decimal and binary and the Hamming cliff problem in binary coding due to the inability to estimate the interval of independent variables [24-26]. As shown in Figure 1, distribution center 1 and distribution center 2 each have two distribution vehicles. Each distribution vehicle performs the distribution task under the constraints of capacity and mileage. For example, the distribution vehicle 1 in distribution center 1 provides distribution services for consumers 4 and 7 , and the distribution route is distribution center $1 \longrightarrow 4 \longrightarrow 7 \longrightarrow$ distribution center 1 .

3.3. Algorithm Steps. In the initialization phase, the algorithm parameters, such as population size and maximum number of iterations, are determined, and the initial position of each individual in the population is initialized. In the specific initialization stage, the MDOVRP is transformed into multiple VRPs by distance clustering, and then, the vehicles are allocated and the driving route is designed.

Genetic evolution strategy: in order to expand the search scope and improve the optimization ability of the solution, the search operator used in this paper is a sequential crossover operator (as shown in Figure 2). Specifically, a gene sequence in parent 1 is randomly selected, and the gene sequence selected by parent 1 is deleted in parent 2 . Child 1 inherits the gene sequence selected by parent 1 , and the remaining genes are copied according to the gene sequence of parent 2 . Similarly, child 2 can be obtained after the transformation and crossover of parent 1 and parent 2. Among them, the elite 


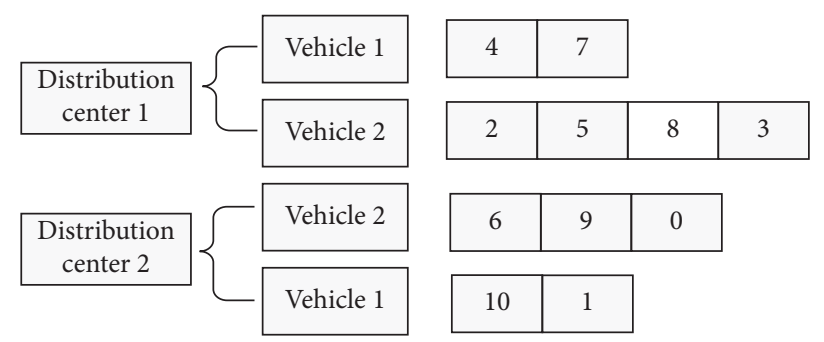

Figure 1: Expression of a solution.

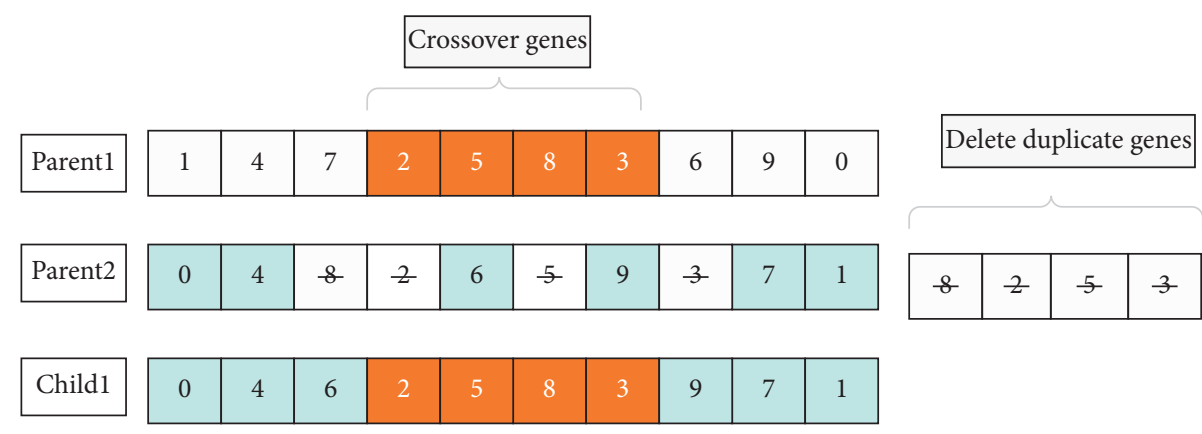

Figure 2: Crossover operations.

principle is introduced in the cross stage; that is, the elite individuals are selected for cross operation through the binary tournament principle. As shown in Figure 2, the genes selected by parent 1 are $2,5,8$, and 3 . The corresponding genes in parent 2 are deleted, and the genes 2, 5, 8, and 3 in parent 1 are obtained by offspring 1 . The remaining genes are inserted in the sequence of genes in parent 2 .

At the same time, in order to improve the diversity of drosophila individuals, three mutation operators, swap, insert, and invert, are used in the algorithm design. Among them, exchange mutation is to randomly select two different locations and then exchange customers in two locations, while shift mutation is to randomly select customers in one location and insert them into another random location. According to the coding method in this paper, the two locations selected by exchange mutation operation and shift mutation operation can be either two locations on the same subpath or two locations on the same subpath It can be two positions on different subpaths, so that different paths can smoothly realize "information exchange" and increase the search scope of solution space. Inversion mutation first randomly selects a subpath (i.e., a distribution route), then randomly selects two different locations on the subpath, and reverses the order of customers between the two locations.

\section{Case Analysis}

In this part, random initialization data are used for numerical experiments. Suppose there are four distribution centers, and each distribution center has two distribution vehicles. The maximum carrying capacity and maximum driving distance of each vehicle are 120 and 400, respectively. The positions of 48 customers are randomly generated in the interval, and the demand of consumers is randomly generated in the interval. The distribution center and customer information are shown in Tables 1 and 2, respectively.

According to Suzuki [19], if $\rho^{0}=1, \rho^{*}=2$; at the same time, Li et al. [21] pointed out that every liter of fuel consumption releases $2.32 \mathrm{~kg}$ of carbon emissions, the unit carbon emission cost is 2 yuan, the daily fixed cost of distribution vehicles is 500 yuan, and the unit variable cost of vehicle distribution is 7.56 yuan.

4.1. Open-Loop and Closed-Loop Distribution. In order to compare the differences between open-loop distribution and closed-loop distribution and to verify the effectiveness of the algorithm, the population 60 is set, the crossover rate and mutation rate are set to 0.9 and 0.1 , respectively, and the number of iterations is 500 . The algorithm is implemented in Java language and run on Intel (R) core (TM) i5-10210U CPU@1.6 GHz, the three algorithms are run 30 times independently, and the optimal results are shown in Figures 3 and 4.

4.2. Multiobjective Solutions. In Figure 5, it can be found that there is an inverse proportional relationship between logistics cost and carbon emission. Therefore, it can be said that, in the actual distribution, the carbon emission of logistics distribution vehicles can be realized by increasing a certain distribution cost; that is, there is a certain turnover 
TABle 1: Depot information.

\begin{tabular}{lccc}
\hline No. & $X$-coor & $Y$-coor & Number of vehicles \\
\hline 1 & 4.159812 & 13.598909 & 2 \\
2 & 21.397101 & 17.109821 & 2 \\
3 & -36.118921 & 42.980109 & 2 \\
4 & 25.498080 & -21.199321 & 2 \\
\hline
\end{tabular}

TABLE 2: Customer information.

\begin{tabular}{|c|c|c|c|}
\hline No. & $X$-coor & $Y$-coor & Demand \\
\hline 1 & -29.729819 & 44.142146 & 10 \\
\hline 2 & -30.659302 & 5.460902 & 9 \\
\hline 3 & 22.639003 & 5.470913 & 17 \\
\hline 4 & -13.169832 & 19.344121 & 6 \\
\hline 5 & -27.409815 & 38.320117 & 11 \\
\hline 6 & 48.897125 & 6.268915 & 3 \\
\hline 7 & 5.237919 & 22.259910 & 15 \\
\hline 8 & -44.987113 & 27.230912 & 18 \\
\hline 9 & -4.178921 & -1.571234 & 15 \\
\hline 10 & 23.029109 & 11.640331 & 15 \\
\hline 11 & 25.276904 & 6.280612 & 20 \\
\hline 12 & -42.620903 & -26.290111 & 7 \\
\hline 13 & -36.667907 & 10.138901 & 10 \\
\hline 14 & -20.668721 & 32.889810 & 8 \\
\hline 15 & -33.039931 & 6.570115 & 15 \\
\hline 16 & -41.379823 & 10.820217 & 20 \\
\hline 17 & -21.940114 & 27.590334 & 13 \\
\hline 18 & -35.188015 & 30.209832 & 15 \\
\hline 19 & 18.601902 & 26.719131 & 12 \\
\hline 20 & -10.938610 & 43.208921 & 14 \\
\hline 21 & -37.760013 & -33.329813 & 22 \\
\hline 22 & 23.769120 & 29.076910 & 12 \\
\hline 23 & -43.029112 & 20.449001 & 9 \\
\hline 24 & -35.299321 & -24.897812 & 17 \\
\hline 25 & -44.759801 & 14.367914 & 10 \\
\hline 26 & -19.328702 & 33.365912 & 10 \\
\hline 27 & 7.397704 & 23.819004 & 16 \\
\hline 28 & 37.397810 & 13.819821 & 18 \\
\hline 29 & -26.624912 & 43.336715 & 21 \\
\hline 30 & -38.559823 & -3.709410 & 10 \\
\hline 31 & -16.779124 & 19.539005 & 12 \\
\hline 32 & -11.559014 & 11.619802 & 17 \\
\hline 33 & -46.547919 & 8.897315 & 17 \\
\hline 34 & 16.228901 & 9.319218 & 23 \\
\hline 35 & 1.789202 & 17.349817 & 15 \\
\hline 36 & -26.398132 & 29.530914 & 9 \\
\hline 37 & 4.348926 & 14.679831 & 13 \\
\hline 38 & -20.670919 & -23.129821 & 18 \\
\hline 39 & -22.826112 & -9.807818 & 10 \\
\hline 40 & -31.097636 & -18.619302 & 17 \\
\hline 41 & -7.848103 & 32.067509 & 17 \\
\hline 42 & 11.876909 & -24.927802 & 20 \\
\hline 43 & -18.927903 & -23.726919 & 26 \\
\hline 44 & -11.918734 & 11.757612 & 13 \\
\hline 45 & 29.839435 & 11.629012 & 20 \\
\hline 46 & 12.267825 & -35.808921 & 17 \\
\hline 47 & -37.927816 & -21.607910 & 19 \\
\hline 48 & 41.983101 & -6.963910 & 12 \\
\hline
\end{tabular}

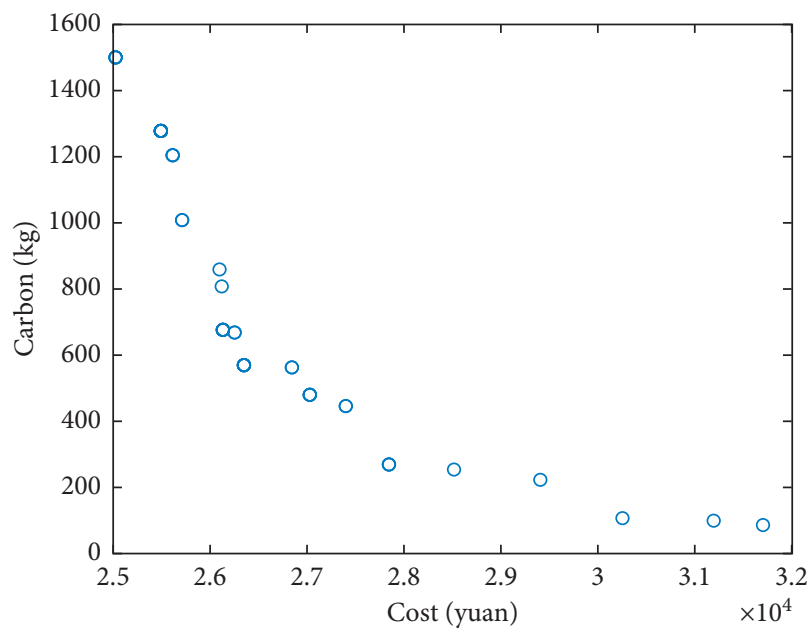

FIgUre 3: Pareto optimality.

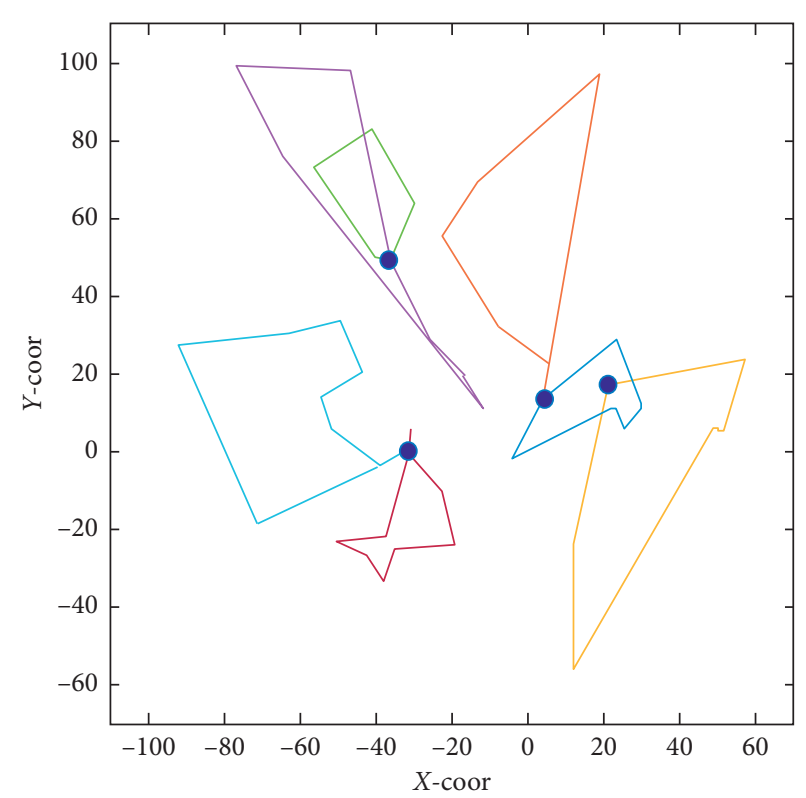

Figure 4: Routes for closed loop.

profit and loss phenomenon between the two. Logistics companies need to weigh the relationship between economic benefits and social benefits when making decisions. It is necessary to realize the unity of economic benefit and social benefit without hindering economic benefit. 


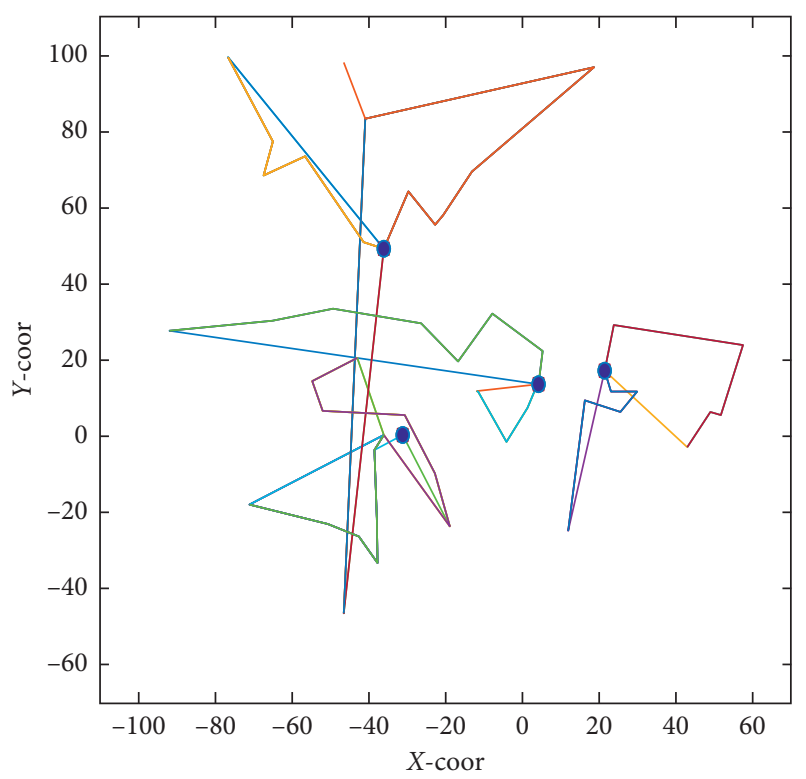

FIGURE 5: Routes for open loop.

\section{Conclusions}

With the rapid development of e-commerce industry, logistics distribution has become the difficulty of express delivery industry. Based on the traditional vehicle routing problem, considering the sharing economy and the hot spot of open-loop distribution, and considering the optimization of carbon emission in logistics distribution, this paper takes the driving distance and load capacity as the key factors to affect carbon emission, establishes a carbon emission model, and puts forward the most effective measures. To solve the dual objective problem of minimizing logistics distribution cost and minimizing logistics carbon emissions, a logistics distribution path planning model with multiple distribution stations is constructed. Aiming at the difficulties of the multiobjective solution and multidistribution station optimization problem coding, a strategy of clustering analysis based on customer distance is designed to schedule distribution vehicles in three steps, which ensures the satisfaction of customers' orders and the smooth operation. The elite crossover principle is used to avoid the solution falling into local optimum. The experimental results show that the solution results of the proposed logistics model and optimization algorithm can take into account the logistics cost and carbon emissions. This study provides theoretical and methodological guidance for the logistics distribution problem considering the openloop distribution problem and carbon emission cost. At the same time, the algorithm also provides a way to solve this kind of NP-hard problem.

The next step is to study the logistics distribution optimization problem with uncertain characteristics and customer return and exchange.

\section{Data Availability}

Data are included in this paper.

\section{Conflicts of Interest}

The authors declare that they have no conflicts of interest.

\section{Acknowledgments}

This work was supported by the Shenzhen Health Economic Society (No. 202014).

\section{References}

[1] Y. He, M. Qi, F. Zhou, and J. Su, "An effective metaheuristic for the last mile delivery with roaming delivery locations and stochastic travel times," Computers \& Industrial Engineering, vol. 145, Article ID 106513, 2020.

[2] L. Schrage, "Formulation and structure of more complex/ realistic routing and scheduling problems," Networks, vol. 11, no. 2, pp. 229-232, 1981.

[3] M. Liu, X. Xu, J. Cao, and D. Zhang, "Integrated planning for public health emergencies: a modified model for controlling H1N1 pandemic," Journal of the Operational Research Society, vol. 71, no. 5, pp. 748-761, 2020.

[4] J. A. Garza-Reyes, B. Villarreal, V. Kumar, and J. DiazRamirez, "A lean-TOC approach for improving emergency medical services (EMS) transport and logistics operations," International Journal of Logistics Research and Applications, vol. 22, no. 3, pp. 253-272, 2019.

[5] Z. Zhang, W. Yao, Y. Wang, C. Long, and X. Fu, "Wuhan and Hubei COVID-19 mortality analysis reveals the critical role of timely supply of medical resources," Journal of Infection, vol. 81, no. 1, pp. 147-178, 2020.

[6] F. Zhou, Y. He, P. Ma, and R. V. Mahto, "Knowledge management practice of medical cloud logistics industry: transportation resource semantic discovery based on ontology modelling," Journal of Intellectual Capital, vol. 22, no. 2, pp. 360-383, 2020.

[7] Y. He, X. Wang, Y. Lin, F. Zhou, and L. Zhou, "Sustainable decision making for joint distribution center location choice," Transportation Research Part D-Transport and Environment, vol. 55, pp. 202-216, 2017.

[8] Y. He, F. Zhou, M. Qi, and X. Wang, "Joint distribution: service paradigm, key technologies and its application in the context of Chinese express industry," International Journal of Logistics Research and Applications, vol. 23, no. 3, pp. 211-227, 2020.

[9] J. Brandão, "A tabu search algorithm for the open vehicle routing problem," European Journal of Operational Research, vol. 157, no. 3, pp. 552-564, 2004.

[10] F. Li, B. Golden, and E. Wasil, "The open vehicle routing problem: algorithms, large-scale test problems, and computational results," Computers \& Operations Research, vol. 34, no. 10, pp. 2918-2930, 2007.

[11] P. P. Repoussis, C. D. Tarantilis, O. Bräysy, and G. Ioannou, "A hybrid evolution strategy for the open vehicle routing problem," Computers \& Operations Research, vol. 37, no. 3, pp. 443-455, 2010.

[12] E. Cao, M. Lai, and H. Yang, "Open vehicle routing problem with demand uncertainty and its robust strategies," Expert Systems With Applications, vol. 41, no. 7, pp. 3569-3575, 2014.

[13] T. Bektaş and G. Laporte, "The pollution-routing problem," Transportation Research Part B-Methodological, vol. 45, no. 8, pp. 1232-1250, 2011.

[14] Y. Xiao and A. Konak, "The heterogeneous green vehicle routing and scheduling problem with time-varying traffic 
congestion," Transportation Research Part E-Logistics and Transportation Review, vol. 88, pp. 146-166, 2016.

[15] T. Woensel, R. Creten, and N. Vandaele, "Managing the environmental externalities of traffic logistics: the issue of emissions," Production and Operations Management, vol. 10, no. 2, pp. 207-223, 2009.

[16] E. Demir, T. Bektaş, and G. Laporte, "A comparative analysis of several vehicle emission models for road freight transportation," Transportation Research Part D: Transport and Environment, vol. 16, no. 5, pp. 347-357, 2011.

[17] Y. Niu, Z. Yang, P. Chen, and J. Xiao, "Optimizing the green open vehicle routing problem with time windows by minimizing comprehensive routing cost," Journal of Cleaner Production, vol. 171, pp. 962-971, 2018.

[18] Y.-J. Kwon, Y.-J. Choi, and D.-H. Lee, "Heterogeneous fixed fleet vehicle routing considering carbon emission," Transportation Research Part D: Transport and Environment, vol. 81, pp. 89-971, 2013.

[19] Y. Suzuki, "A dual-objective metaheuristic approach to solve practical pollution routing problem," International Journal of Production Economics, vol. 176, pp. 143-153, 2017.

[20] E. Jabir, V. V. Panicker, and R. Sridharan, "Design and development of a hybrid ant colony-variable neighbourhood search algorithm for a multi-depot green vehicle routing problem," Transportation Research Part D: Transport and Environment, vol. 57, pp. 422-457, 2017.

[21] J. Li, D. Wang, and J. Zhang, "Heterogeneous fixed fleet vehicle routing problem based on fuel and carbon emissions," Journal of Cleaner Production, vol. 201, pp. 896-908, 2018.

[22] K. Deb, A. Pratap, S. Agarwal, and T. Meyarivan, "A fast and elitist multi-objective genetic algorithm: NSGA-II," IEEE Transactions on Evolutionary Computation, vol. 6, no. 2, pp. 182-197, 2002.

[23] H. Rai and A. Yadav, "Iris recognition using combined support vector machine and hamming distance approach," Expert Systems With Applications, vol. 41, no. 2, pp. 588-593, 2014.

[24] Y. He, X. Wang, F. Zhou, and Y. Lin, "Dynamic vehicle routing problem considering simultaneous dual services in the last mile delivery," Kybernetes, vol. 49, no. 4, pp. 1267-1284, 2019.

[25] F. Zhou, Y. He, and L. Zhou, "Last mile delivery with stochastic travel times considering dual services," IEEE Access, vol. 7, pp. 159013-159021, 2019.

[26] F. Zhou, M. K. Lim, Y. He, and S. Pratap, "What attracts vehicle consumers' buying: a saaty scale-based VIKOR (SSCVIKOR) approach from after-sales textual perspective?" Industrial Management and Data Systems, vol. 120, no. 1, pp. 57-78, 2019. 